\title{
Klinische, bakteriologische und mikroskopische Befunde bei der Verwendung des Radiumbromids in der Therapie der Hautkrankheiten.
}

\author{
Von \\ Dr. Kurt Strassmann, \\ Spezialarzt fur Hautkrankheiten, Kattowitz 0./S., ehem. Assistenzarzt a. đ. Kgl. dermat. \\ Klinik.
}

Im Jabre 1903 ging aus der hiesigen Klinik eine Arbeit Halkins hervor, die, wie meine nachstehenden Mitteilungen den Einfluß der Becquerelstrahlen auf die Haut behandelte.

Für seine experimentellen Untersuchungen wählte $\mathrm{Hal} \mathrm{k}$ i n neben Kaninchen- besonders Scbweinehaut, deren Struktur der menschlichen sehr nahe kommt. In methodischer Reihenfolge wurden von ihm auf beiden Seiten des Tieres stets je eine Stelle in gleicher Weise 1 bis 2 Stunden mit Radium behandelt, um so gleichwertige Beobachtungsobjekte für makroskopische und mikroskopische Untersuchung zu gewinnen.

Mikroskopisch zeigten sich nach 1 Stunde Belichtung bereits nach 3 mal 24 Stunden Veränderungen, die täglich an Intensität zunahmen und sich am Gefäßapparat abspielten. Die Kapillaren waren erweitert und deutlicher sichtbar. Am 7. Tage machte sich bereits eine mäßige Infiltration um die Gefäße bemerkbar. Das ganze Kapillarnetz war strotzend mit Blut gefüllt, die Endothelzellen gequollen und ihre Kerne voluminöser. Nach zweistündiger Bestrahlung traten die mikroskopischen Veränderungen zeitlich nicht früher auf.

$\mathrm{Makroskopisch}$ dagegen trat nach einstündiger Belichtung erst am 15. Tage die 1. Reaktion in Form einer circumscripten lividen Verfärbung zu Tage, welche nach zweistündiger Applikation bereits am 8. Tage sichtbar wurde und einen lebhatteren Farbenton annahm. In der 2. und 3. Woche bedeckten sich die durch 1-2stündiger Bestrahlung gewonnenen Reaktionsherde unter Pigmentation mit Schuppen. 
In der Zwischenzeit waren die mikroskopischen Veränderungen immer weiter vorgeschritten. In den Endothelzellen trat Vacuolenbildung anf. Die gleichen Degenerationserscheinungen machten sich jetzt auch an den Bindegewebszellen und an den Epithelien bemerkbar. Am 21. Tage etwa war die Hornschicht aufgelockert und abgehoben. Die Zellen schienen in ihrem Zusammenhang gelockert, die Zwischenräume größer geworden, die Zellen selbst noch stärker gequollen und väcuolisiert. Hier und da fand man bereits zerstreut zu Grunde gegangene Zellen- und Kernfragmente. Die Kapillaren waren zu großen Bluträumen erweitert, die Intimazellen sprangen in das Gefäblumen vor. Allenthalben Hämorrhagien, Infiltration und Blutpigmentanhäufung. Über den mikroskopischen Abheilungsprozeb hat $\mathrm{H}$ a lk in keine Untersuchungen angestellt. Den makroskopischen Heilungsverlauf stellte er etwa wie den Ablauf einer sublutanen hämorrhagisehen Entzündung dar. Der Abheilungsbeginn ist in die 4.-5. Woche zu verlegen.

Auf Grund dieser an $\mathbf{n}$ ormale $\mathrm{r} \mathbf{S} \mathrm{ch}$ we in e ha $\mathrm{u}$ t erhaltenen Befunde glaubte auch $\mathrm{Ha} 1 \mathrm{kin}$ pathologische Prozesse durch Radium günstig beeinflussen zu können. Er dehnte nach dem Vorbild von Dantes und Bloch, die nach Erzeugung von Ulzeration eine Heilung des Lupus erythematodes mit Narbenbildung beobachtet hatten, seine Versuchsreihen nun auch auf $L$ u p us $\vee$ u $l g$ aris aus. Auf Grund von 7 Beobachtungen und nach Applikationen von $6 \mathrm{mal} 30$ Minuten bis zu 14 mal 4 Stunden kam Halk in schließlich zu dem Ergebnis, dah das Radium in der Therapie des Lupus vulgaris keine Verwendung finden könne, weil die Sehädigung der Gewebe und die Ulzerationen, die durch dasselbe gesetzt werden, in keinem Verhältnis zum therapeutischen Erfolge ständen: „Der Lupusherd in der Tiefe ist gänzlich unverändert geblieben und erweckt den Eindruck, als wenn die Tuberkel mit ihren zahlreichen Riesenzellen von den radio-aktiven Strahlen gar nicht getroffen worden seien." Es fand also eine Beeinflussung des Lupus lediglich nur durch die ulzeröse Zerstörung der Gewebe statt.

Für diese Versuche standen $\mathrm{Halk}$ in damals $0.13 \mathrm{~g}$ Ra d i u mB a r y u m-B r o m i d zar Verfügung, das in einer Metallkapsel, die mit einem $0.1 \mathrm{~mm}$ dicken Aluminiumdeckel versehen war, zur Verwendung kam.

Nachdem sich nunmehr herausgestellt, daß das Baryum als Verunreinigung die Wirkung des Präparates wesentlich beeinträchtige, gelang es Becquerel, Curie und Giesel durch Abspaltung des Baryums $r$ eines $\mathrm{R}$ a di $\mathrm{um}$ b r o m i d herzustellen, das dem alten Präparat bedeutend überlegen ist.

Dank der Güte des Herrn Prof. Giesel erhielten wir (aus der Chininfabrik Buchler \& Co., Braunschweig) von diesem von Baryum befreiten reinen Radiumbromidpräparat 3 mal je $10 \mathrm{~mm}$ enthaltende Hartgummikapseln. Diese besitzen einen etwa $21 / 9 \mathrm{~mm}$ Radius messenden, im Zentrum gelegenen Ausschnitt, der durch ein Glimmerplättchen nach außen abge- 
Klin., bakt. u. mikr. Bef. b. d. Verw. d. Radiumbromids etc. 421

schlossen ist. Unter diesem befinden sich in einer kleinen Aushöhlung die Krystalle. Abgeschlossen ist das Glimmerplättchen an der Peripherie durch einen Paraffinring. Dieser letztere wird durch 2 fest aufeinander verschraubbare Kapselteile fixiert.

Das Präparat wurde stets in der Weise appliziert, daß das mit Glimmerplättchen abgeschlossene Zentrum mit dem zu bestrahlenden Herde korrespondierte. Die Kapsel selbst wurde durch einen Heftpflasterstreifen auf die Haut fixiert.

So einfach nun auch diese Technik sich gestaltet, stößt doch die Behandlung der Präparate selbst auf recht erhebliche Schwierigkeiten, da das hygroskopische Radiumbromid durch Feuchtigkeitsaufnahme an Wirkung einbiußt. Ein zeitweiliger Aufenthalt im Exikator konnte diesen Übelstand nicht beheben. Im Gegenteil ließ sich stets nachher am Fluorescenzschirm und an der Form der Reaktion eine Herabsetzung der Wirkungsintensität des Radiums nachweisen. Bei verschiedenen von uns ausprobierten chemischen Austrocknungs-Methoden war stets die gleiche schädigende Wirkung auf das Radium zu konstatieren. Um diese Feuchtigkeitsaufnahme zu verhindern, wurden schließlich die Kapseln mit Gummik ond oms umhüllt und dann den erkrankten Stellen aufgelegt. Bei Manipulationen in der Mundhöhle und bei nässenden Geschwürsflächen wurden 2-3 Kondoms kreuzweise über einander gestülpt.

\section{Klinische Beobachtungen.}

Die ersten Mitteilungen hierüber stammen von $W$ a l k off and Giesel, die unter dem Einfluß ihrer Präparate an normaler Haut einige Tage nach der Einwirkung entzündliche Rötung auftreten sahen, die schließlich zur Ulzeration führte und mit Narben abheilte. Ganz ähnliche Befunde konnte $\mathrm{Asch}$ kin a s erbeben, der nach zweistündiger Applikation mehrere Tage später die 1. Reaktion in Form einer entzündlichen Röte beobachtete, die nach 30 Tagen ihren Höhepunkt erreichte. Becquerel und $\mathrm{Cu}$ rie bestätigten diese Beobachtungen. $\mathrm{Cu}$ i e sah nach zehnstündiger Applikation am eigenen Arme kurz nach der Bestrahlung eine Rötung und erst nach 52 Tagen eine Ulzeration auftreten.

Diesen letzteren Befunden kommen meine Beobachtungen am nächsten, die zum Teil ebenfalls an der eigenen gesunden Haut gemacht wurden. Sie unterscheiden sich jedoch von allen anderen durch die Schnelligkeit des Reaktionseintrittes und dessen Intensität.

Die Stärkeder erzeugten Reaktionstebt nun im direkten Verbältnis zur Expositionsdauer. Außerdem 
aber ist von Bedeutung, ob man das Präparat in einer Sitzung auf die Gewebe einwirken läßt, oder in e inzelne $\mathrm{n}, \mathrm{d} u \mathrm{rch}$ kürzere oder längere Pausen unterbrochenen Applikationen.

\section{Resuitate nach einmaliger Bestrahlung.}

Nach einer Expositionsdauer von $3 \mathrm{Min}$. wird eine circumscripte fast unmittelbar nach dem Versuch zu konstatierende rosa-rote Verfärbung bemerkbar, die dem Ausschnitt der Kapsel entspricht. Ob diese wenige Stunden dauernde primäre Rötung schon einer direkten Gefäßbeeinflussung zuzuschreiben ist, läßt sich nicht entscheiden. Nach ca. 24 Stunden nimmt derselbe Herd eine deutliche entzändliche Röte an, die in den nächsten 2 Tagen sich ständig verstärkt. Gewöhnlich hält der entzündliche ProzeB ca 1-2 Wochen an, um dann allmählich wieder abzulaufen. Doch zeigt der Herd, wo das Präparat gelegen, noch nach ca 6 Monaten einen pigmentierten schuppenden Fleck mit Haarausfall und leichter oberflächlicher Epithelalteration, bis auch er schließlich nach etwa 8 Monaten wieder seine Normalbeschaffenheit annimmt.

Verlängert man die Expositionsdauer über 3 Min. hinaus, so unterscheiden sich die Reaktionen nach einer einmaligen Exposition von 5-10 Min. dadurch, daß die entzündliche Röte in der gleichen Zeit einen intensiveren Farbenton annimmt, ohne dak sie deshalb eher zu Tage trete. In entsprechender Weise gilt dies auch von den Expositionen nach 20-30 Min. - Nach einstündiger Behandlung gesellt sich zu der etwa in derselben Zeit auftretenden entzündlichen Röte auch eine Sehwellung. Scharf hebt sich der Reaktionsherd, der genau dem Kapselausschnitt entspricht, von der Umgebung ab und ist leicht über das Niveau der Haut erhaben. In unmittelbarer Nachbarschaft scheint also die Haut von den Strahlen nicht getroffen zu sein. Am 3.-4. Tage entwickelt sich eine Blase und am 5. eine oberflächliche Erosion. - Nach 6-12 Stunden kehrt dasselbe Bild im allgemeinen wieder, doch schließt sich dem Auftreten der Erosion eine U l z e ration an. So lassen sich nach fortgesetzter Behandlung bei Applikationszeiten von $24,48,60$ und 120 Stunden bis z u ca. $1 / 2 \mathrm{~cm}$ ti ef e G e$\mathrm{schw}$ üre erzeugen. Hieraus geht also hervor, daß im Auftreten der entzündlichen Rötung kaum nennenswerte Differenzen sich zeigen. Bei dem A uftreten der Ulzeration dagegen machen sich nach den über 10 Stunden hinausgehenden Sitzungen geringe Unterschiede insofern bemerkbar, als die Ulzeration entsprechend der längeren Bestrahlungsdaner früher eintritt, doch gehen diese Unterschiede nicht über $1 / 2-1$ Tag hinaus.

Ähnlich liegen auch die Verhältnisse bezüglich des Fortschreitens des ulzerierenden Prozesses, der um so intensivere Formen annimmt, je länger das Präparat wirken konnte.

Entsprechend dem wechselnden Grade des erzielten Effektes wechselt nun auch die Ausheilungsdauer. Die 
akuten Entzündungserscheinungen, wie wir sie als allererste Reaktion nach 3-5 Minuten langer Bestrahlungsdauer beobachten, verschwinden gewöhnlich nach 1-4, nach einer Bestrahlungsdauer bis zu 30 Min. nach 6-8 Wochen. Die rückständige Pigmentation ist noch nach Monaten sichtbar. - Oberflächliche Erosionen gehen bereits nach 12-14 Tagen eine Restitution ein. Die entzündliche Röte blaßt nach $6-8$ Wochen unter Zurücklassung einer weißen, glatten, etwas atrophischen Stelle ab. Diese im kutanen Bindegewebe sich abspielende Atrophie ist durch eine mehr oder minder hochgradige Degenerationserscheinung im subkutanen Bindegewebe zu erklären. - Die Ulzerationen, die einer Bestrahlungsdauer von etwa 6-24 Stunden entsprechen, zeigen nach ihrer vollständigen Ausbildung ähnlich den Röntgenulzera einen diphtherischen gelben, stark adhärenten Belag. Die makroskopisch sichtbare, reaktive, zur Abstoßung führende Fntzändung läßt oft 1-2 Monate auf sich warten. Die totale Abheilung erfolgt erst nach otwa 2-3 Monaten. Bei einer Bestrahlungsdauer von 24-60 Stunden reinigen sich dieselben erst nach 2-3 Monaten und heilen nach etwa 3-4 Monaten ab.

Bei allen diesen Formen der Reaktionen sind die subjektiven Beschwerden auffälig gering und werden selbst bei Ulzerationen nur als zeitweise auftretendes Jucken und Brennen angegeben.

\section{Resultate nach mehreren, in längeren oder kürzeren Pausen unterbrochenen Sitzungen.}

Anders liegen die Verhältnisse, sobald wir die gleichen Expositionszeitenauf mehrere entsprechend kürzere Sitzungen verteilen. Hierbei sind dann wieder verschiedene Abstufungen zu unterscheiden, je nach der Länge der zwischen den einzelnen Dosierungen eingeschalteten Ruhepausen. Je schneller die Applikationen auf einander folgen, desto eher und energischer tritt die Reaktion ein. Während wir, wie erwähnt, nach einmaliger einstündiger Einwirkung bereits uach 5 Tagen eine Erosion beobachten, erzielen wir in derselben Zeit nur eine Blasenbildung, wenn wir diese Dosis in 2 Tagen auf $2 \times 30$ Minuten verteilen. Erst erheblich stärkere Applikationen von $5 \times 30$ Minuten führen wieder in 8 Tagen zu einer Erosion.

Noch frappanter wird dieser Unterschied, wenn wir dieselben Versuche mit $z$ weitägigen Pausen ausführen, indem so eine Erosion überhaupt nicht zu stande kommt.

Mankann also ganz nach Wunsch und Belieben die mannigfachsten Modifikationen erzielen, wie aus nachstehender Tabelle ersichtlich ist. Die in den Kolumnen angegebenen Stundenzahlen bedeuten den Zeitpunkt, an dem die betreffende Reaktion sichtbar wurde. Die römischen Zahlen deuten ihre verschiedenen Formen an, und zwar wollen wir unter I.: eine leichte entzündliche Röte; II. : ein intensives Rot; III.: eine Blasenbildung; IV.: eine Erosion; 
V.: eine Ulzeration verstehen. Innerbalb der einzelnen Kolumnen bestehen gewisse Unterschiede insofern, als entsprechend der längeren Exposition staffelförmig allmählich immer lebhaftere Reaktionen auftreten.

Tabelle I.

\begin{tabular}{|c|c|c|c|c|c|}
\hline $\begin{array}{l}\text { Expos.- } \\
\text { Zeiten } \\
\end{array}$ & I & II & III & IV & $\nabla$ \\
\hline 3Min. & ca. 24 Std. & 60 Std. & - & - & - \\
\hline 10 & $\mid \begin{array}{c}24 \text { Std. intensiv. } \\
\text { Farbenton }\end{array}$ & $n$ & $n$ & $\eta$ & $"$ \\
\hline 20 & $n$ & stärk. Rot & $n$ & $n$ & $n$ \\
\hline $60 n$ & $n$ & intens. Rot mit & 90 Std. & $n$ & 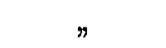 \\
\hline $6 \mathrm{St}$ & $\begin{array}{c}18 \text { Std. lebhaftes } \\
\text { Rot }\end{array} \mid$ & Schwell. 48 st. & $n$ & $120 \mathrm{Std}$ & $136 \mathrm{std}$ \\
\hline $12 n$ & $n$ & „36 St. & $\begin{array}{l}\text { erhaben ut leicht } \\
\text { platzend. } 60 \mathrm{Std} \text {. }\end{array}$ & 96 Std. & 120 std. \\
\hline 24 & noch int. Rot & $n$ & $n$ & int. Zerfall & int. Zerfall \\
\hline $48 \%$ & $n$ & std. zunehm. & $n$ & $\eta$ & r \\
\hline $96 n$ & $"$ & $"$ & $n$ & $\eta$ & $n$ \\
\hline 120 & \# & $n$ & $\eta$ & & $\eta$ \\
\hline
\end{tabular}

Tabelle II.

Expositionen von täglich wiederholten Dosierungen.

\begin{tabular}{|c|c|c|c|c|c|}
\hline & I & II & III & IV & V \\
\hline $5 \times 3$ & 30 Std. & 48 Std. & - & - & - \\
\hline $3 \times 10^{\stackrel{9}{\rightleftarrows}}$ & $n$ & 36 Std. & - & - & - \\
\hline $2 \times 30$. & 24 Std. & " & $120 \mathrm{Std}$. & - & - \\
\hline $5 \times 30^{7}$ & $"$ & $n$ & $\pi$ & 192 Std. & - \\
\hline
\end{tabular}

Tabelle III.

Expositionen von 3-30 Min., die in zweitägigeu Pausen wiederholt wurden.

\begin{tabular}{|c|c|c|c|c|c|}
\hline & I & II & III & IV & V \\
\hline $5 \times 3$ & $24 \mathrm{Std}$ & 60 Std. & - & - & - \\
\hline 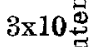 & $n$ & $"$ & - & - & 一 \\
\hline $2 \times 30$. & intensiv. Rot & 48 St int. Rot & 288 St. & - & - \\
\hline $5 \times 30^{z}$ & $n$ & $n$ & - & - & - \\
\hline
\end{tabular}


Klin., bakt. u. mikr. Bef. b. d. Verw. d. Radiumbromids eto. 425

Fassen wir dies alles zusammen, so ergibt sich für die therapeutische Verwendung des Radiums der Hinweis, daß es zweckmäßiger ist, lange Pausen zwischen nicht zu kurzen Expositionen einzuschieben. Holzknecht rät sogar, jede einzelne Reaktion erst ablaufen zu lassen, ehe man zur nächsten Dosierung schreitet. Nur stößt diese Form in der Pruxis bei den Patienten wegen Zeitmangel häufig auf unüberwindliche Hindernisse. Um diese Schwierigkeit zu umgehen, applizieren wir auf Grund zahlreicher Untersuchungen und Beobachtungen die Kapsel meist in 3-5 Sitzungen 1-2 mal täglich. Bezüglich der Dauer der einzelnen Sitzungen verweise ich auf die Behandlung der einzelnen Affektionen.

Es sei nur noch ausdrücklich hervorgehoben, daß alle meine Zeitangaben keinen absoluten Wert beanspruchen können. Erstens wissen wir, daß eine ganze Reihe verschiedenartiger Radiumpräparate auf den Markt gebracht werden, die wegen ihrer Verunreinigungenkeineswegs gleichwertig sind. Nach Becquerel, Curie, Halkin, Giesel wenigstens gehen die Angaben hierüber weit auseinander. Bohn erzielte mit seinem Präparate, gemessen am Chromoradiometer nach $\mathrm{Holzknecht}$, in 12 Stunden 8 H.-Einheiten, ein Resultat, zu dem wir bereits im 12. Teil der Zeit gelangten. Vergleichende Beobachtungen des Halkinschen Präparates mit unseren neuen ergaben ganz in die Augen fallende Unterschiede, die sich am Fluoreszenzschirm, und, wie ja aus unseren eben erwähnten Versuchen hervorgeht, auch durch die Reaktion auf der Haut nachweisen ließen.

Außerdem haben wir an unseren Präparaten feststellen können, daß die Strahlungsintensität ein und desselben Präparates wechselt. Wir glauben diese Differenzen auf die, wie schon oben erwähnt, nicht absolut vermeidbare Feuchtigkeitsaufnahme zurückführen zu müssen.

Als Beleg für diese Tatsachen möge beifolgende, am Holzknechtschen Chromoradiometer angestellte Versuchsreihe gelten:

Es wurden in einmaligen Sitzungen die Kapseln den von $\mathrm{Holz}$ knecht angegebenen Reagenskörpern aufgelegt und dann während der 
in der Tabelle genannten Zeiten die Reaktionen an der Standard-Skala zum Chromoradiometer verglichen und abgelesen. "H." bedeutet die hierfür von $\mathrm{Holzknecht} \mathrm{eingeführte} \mathrm{Einheit.}$

\begin{tabular}{|c|c|c|c|}
\hline $\begin{array}{l}\text { Expositions- } \\
\text { Zeiten }\end{array}$ & Ä1testes Präparat & II. Präparat & Jüngstes Präparat \\
\hline 2 Minuten & Keine Reaktion & Keine Reaktion & Keine Reaktion \\
\hline 3 & 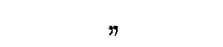 & $n$ & 3. (4) H. \\
\hline 5 & $"$ & $3 \mathrm{H}$. & $n$ \\
\hline 10 & $n$ & $n$ & $"$ \\
\hline 15 & $3 \mathrm{H}$. & $"$ & $"$ \\
\hline 20 & $"$ & $"$ & $4 \mathrm{H}$ \\
\hline 25 & 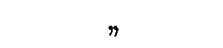 & $n$ & $\eta$ \\
\hline 30 & $"$ & $4 \mathrm{H}$ & $5 \mathrm{H}$. \\
\hline 1 Stunde & $n$ & $6 \mathrm{H}$. & $8 \mathrm{H}$ \\
\hline 6 Stunden & $6 \mathrm{H}$ & $8 \mathrm{H}$ & $20 \mathrm{H}$ \\
\hline $12 n$ & (12) $16 \mathrm{H}$. & (24) $20 \mathrm{H}$. & $24 \mathrm{H}$. und mehr \\
\hline
\end{tabular}

\section{Beobachtungen an Hautkrankheiten.}

Die Tatsache (Bohn, B ecquerel, Caspari, Danysz, Hartigan), daß, wie nach Röntgen, auch bei Radiumbehandlung pathologische Gewebe eher und intensiver reagieren, als normale $\mathrm{Haut}$, fand auch durch unsere folgenden Beobachtungen Bestätigung.

Lichen ruber planus. (Vier Fälle.)

Zwei Fälle von disseminiertem Ruber planus, lokalisiert am Abdomen und an den Beugeseiten der Extremitäten. Typische Lichen ruber planus-Knötchen dicht agregiert stehend.

Es kamen zur Bestrahlung 12 Stellen, zum Teil mehr einzeln stehende Knötchen, zum Teil beetartig agregierte Effloreszenzen.

Nach drei Sitzungen von je 5 Minuten in eintägigen Intervallen trat am 6. Tage nach Beginn der Behandlung eine akute entzündliche Rötung der Knötchen und deren direkter Umgebung ein. Diese Rötung bestand durch 2 Wochen. Nach 14 Tagen blaßte die entzündliche Rötung ab, ohne daB die Effloreszenzen selbst irgendwelche Beeinflus sung zeigten.

Dieselben Herde wurden in der 3. Woche nach der ersten Behandlung nochmals einer gleichen Bestrahlungsperiode unterworfen. Der Verlauf und Effekt war der gleiche. 
Klin., bakt. u. mikr. Bef. b. d. Verw. d. Radiumbromids etc.

In der 6. Woche nach Behandlungsbeginn wurde zum dritten Male in gleicher Weise bestrahlt. Hier trat nach den bereits beschriebenen Anfangserseheinungen mit dem Abblassen der Rötung eine leichte und dauernde Abflachung der bestrablten Knötchen ein.

Zwei Fälle von $L i c h e n$ ruber verrucosus am Unterschenkel.

Die verrucösen Herde (6 Stellen) wurden derselben Bestrahlung wie oben unterworfen. Es traten dieseiben Reaktionserscheinungen erst nach dem dritten Behanalungsturnus anf; gleichzeitig mit dem Abblassen. der Reaktion entstand eine matte, weißgraue Verfärbung der Effloreszenzen und ein leichtes Abflachen derselben. Der papilläre Charakter verschwand, während die Knötchen selbst bestehen blieben.

Drei Stellen worden einer einmaligen Bestrahlung von 15 bis 30 Minuten unterworfen. Daraus resultierten die gleichen Reaktionserscheinungen. Eine Beeinflussung des Herdes nach Abblassen der Reaktion ließ sich nicht konstatieren.

Drei Stellen wurden dreimal mit je eintägigen Intervallen einer Bestrahlung von 15 Minuten ausgesetzt. Am 2. Tage Auftreten von Entzündungsreaktionen, am 4. bis 5. Tage oberflächliche Erosionen und dann eine Ulzeration, die nach 14 Tagen mit einer zarten weißen Narbe abheilte.

Die Effloreszenz selbst war vollständig verschwunden. Eine Abbeilung war also nur durch das Herbeiführen einer Ulzeration zu erreichen.

\section{Lupus erythematodes.}

Da sich die Erkrankungsfälle der Bestrahlung gegenüber sehr verschieden zeigten, muß ich dieselben einzeln anführen:

I. Fall: Lokalisiert an der Nase und den angrenzenden Wangenteilen. Chronisch langsam progrediente Form mit narbigem Zentrum und rotem, etwas erhabenem Rande.

Der Rand wurde fortlaufend täglich (jede Stelle) 5 Minuten bestrahlt. Diese Bestrahlung wurde mit eintägigen Intervallen $3 \mathrm{mal}$ wiederholt. Am Ende der 1. Woche eine typische reaktive Entzündung, die im Laufe der 2. Woche abblaßte, ohne Beeinflus ung des $\mathrm{H}$ e $\mathrm{r}$ d e s.

Am Ende der 3. Woche wurde derselbe Bestrahlungsturnus wiederholt. 8 Tage später (4. Woche) trat Blasenbildung auf. Nach Abhebung der Blasendecke und Ablauf der Reaktion in ca. 1 Woche zeigte sich ein negatives Resultat.

Am Ende der 5. Woche Wiederholung des obigen Bestrahlungsturnus. 5 Tage später (6. Woche) oberfächliche Erosion. Nach 14 Tagen (8. Woche) Abheilen derselben und des Erkrankungsherdes selbst mit einer zarten weißen Narbe.

II. Fall: Ebenfalls an Nase und angrenzenden Wangenteilen lokalisiert. Akutere Form mit breitem Entzündungswall. An 3 Tagen 
wurde je einmal 5 Minuten jede Stelle des Randes bestrahlt. Nach Ablauf der 1. Woche keine Reaktion. Am 7.-11. Tage viermalige Bestrablung jeder Stelle zu 5 Minuten. Am 14. Tage oberflächliche Erosion. Am 26. Tage Epithelisierung und langsame Abheilung mit einer oberflächlichen Narbe. Damit verschwindet der entzüudliche Wall selbst.

1II. Fall: Etwas abseits hiervon steht ein Fall von Lupus erythematodes des Gesichts (Nase und Wangen), der einige Jahre vorher mit Röntgenstrahlen behandelt worden war, mit dem Ergebnis, daß damals die ulzerierten Stellen mit weißer Narbe abheilten, während der größere Teil der Affektion vollständig unbeeinflußt blieb. Späterhin bildeten sich in der durch die Behandlung im ganzen narbig verdickten Haut zahlreiehe Gefäßektasien. - Viermalige Radium-Bestrahlung in täglichen Dosierungen zu je 5 Minuten ließen keine Reaktion aufkommen. Das gleiche negative Ergebnis uach fünfmaligem Bestrahlen derselben Stellen zu je 10 Minuten. Wurden nun die täglichen Sitzungen an den gleichen Herden auf 5 mal 15 Minuten ausgedehnt, so entwickelte sich am 7. Tage eine Erosion, die erst drei Wochen später sich epithelisierte. Mit dem Auftreten der Narbe verschwanden auch die Gefäßektasien im nahen Bezirk. Es gelang überhaupt niemals, ohne Frosion in subkutaner Narbenbildung die GefäBektasien zum Verschwinden zu bringen.

\section{Gefäßektasien auf Röntgennarben.}

4 Fälle $\vee$ on L u p u v ulgaris; fünftägige Bestrahlung jedes einzelnen Herdes zu je 5 Minuten zeitigten keine Reaktionen. In der gleichen Weise verliefen die gleichen Sitzungen bis zu 10 Minuten und auch 15 Minuten fruchtlos. Nach 5 mal 20 Minuten entwickelte sich eine oberflächiche Erosion, der keine entzïndliche Reaktion vorausging. Erstere epithelisieren sich 4 Wochen später. ohne daß die Gefäßektasien verschwinden. 2 malige Bestrahlungen zu je 5 Minuten in zweitägigen Pausen verabreicht, brachten weder eine Reaktion des Gewebes noch eine Veränderung an den Gefäßen zu stande.

\section{Naevi vasculosi.}

Über die erfolgreiche Behandlung dieser Affektion liegen

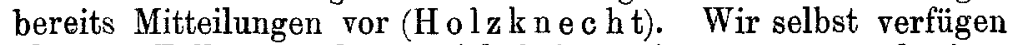
über 12 Fälle, von denen sich bei 8 eine augenscheinliche Besserung bezw. Heilung konstatieren ließ. Die übrigen entzogen sich weiteren Beobachtungen, oder stehen noch in Behandlung. In sämtlichen Fällen handelte es sich um oberflächliche Feuermäler von Erbsen- bis Kleinhandtellergröße im Gesicht und am Halse. 
Klin., bakt. u. mikr. Bef. b. d. Verw. d. Radiumbromids etc. 429

Einmalige Sitzungen ron 5 Minuten zeitigten nach 5 Tagen eine entzündliche Röte, nach deren Rückgang in der zweiten und dritten Woche der Naevus selbst blasser wurde. Das gleiche Resultat nach einmaliger Bestrahlung von 10 Minuten. Dieser günstige Erfolg war allerdings nur von kurzer Dauer, da das Feuermal etwa in der 4. Woche wieder seinen alten Farbenton annahm. - Tägljche Bestrahlungen von 4 mal 5 Minuten führten am 5. Tage zu einer hochgradigen reaktiven Entzündung, auf der sich am 8.-10. Tage eine Blase bildete. Nach der Abbeilung in der 3. Woche zeigte sich ebenfalls nur ein vorübergehendes Blasserwerden. Wurde der gleiche Bestrahlungsturnus auf täglich 5 mal 10 Minuten ausgedehnt, so heilte die am 8. Tage auftretende Erosion 14 Tage später mit einer milchweißen Narbe ab. Am zweckmäßigsten erwies sich der Behandlungsmodus von $4 \mathrm{mal} 5$ Minuten mit zweitägigen Intervallen. Die hierbei auftretende entzündliche Röte hielt sich in engen Schranken, and ohne nachweisliche Epithelalterationen heilte in der 7. Woche der Naevus mit einer kaum wahrnehmbaren subkutanen $\mathrm{Narbe}$ a b. In 2 Fällen wurde wegen eines Rezidivs die gleiche Maßnahme wiederholt. Bis jetzt (6 Monate später) noch kein Rückfall.

\section{Naevi pigmentosi.}

Bei einem neugeborenen Kinde wurden 4, etwa fünfpfenniggroße Naevi pigmentosi pilosi bestrahlt. Dieser Fall ist um so bemerkenswerter, als die Abheilung zugleich mit einer Beeinflussung der Hypertrichosis einhergeht. (J. Macintyre, Dany s.) Mit gleich gutem Erfolge wurde bei einem 19jährigen Mädchen ein Leberfleck am rechten äußeren Augenwinkel and dem unteren Lidrande behandelt. Zur Vermeidung der hierbei stets auftretenden Conjunctivitis ( $\mathrm{Himsted}$ und Nagel, Crzellitzer) wurde der Lidrand durch Heftpflaster abgezogen und an der Wange fixiert. Nach täglichen Applikationen von viermal 15 Min. reagierte der bestrahlte Herd am 5. Tage mit einer entzündlichen Röte, der am 7. Tage eine Erosion folgte. Nach ihrer Abheilung in der 3. Woche verschwand auch der $\mathrm{Naevus} \mathrm{mit} \mathrm{Zurücklassung} \mathrm{einer}$ Narbe. Viermalige Dosierungen ron 5 Min. mit zweitägigen Pausen führien nach 12 Tagen zu einer entzündlichen Röte, mit deren Abblassen am Ende der 3. Woche auch der Naevus abheilte, ohne daß es zu einer Erosion und Narbenbildung gekommen wäre.

\section{Acne rosacea.}

Auch hierïber liegen bereits Mitteilungen mit erfolgreicher Behandlung vor. (Torök und Schein, J. Macintyre.) Bei den von uns beobachteten Fällen handelte es sich um eine 
hochgradige Schwellung und Rötung der Nase mit Knötchen-, Pustelbildung und GefäßBektasien. Tägliche Dosierung von viermal $10 \mathrm{Min}$. führten bereits am 4. Tage zu einer entzündlichen Röte, die deutlich gegen die blaurote Umgebung hervortrat. Am 4. Tage Schwellung, am 6 . Tage hob sich die Epitheldecke $\mathrm{ab}$, und zweimal 24 Stunden später oberflächliche Erosionen. Abheilung nach 4 Wochen mit einer kaum erkennbaren subkutanen Narbe. Wurden die gleichen Dosierungen mit zweitägigen Pausen verabreicht, so kam es nur noch zu einer entzïndlichen Rötung am 12. Tage, und zu einer Besserung, deren kosmetisches Resultat nach Wiederholung der gleichen Prozedur 2 Monate später noch erhöht wurde. Gefäßektasien heilten nur bei gleichzeitiger Narbenbildung ab und ließen sich durch anderen Behandlungsmodus nicht beeinflussen.

\section{Psoriasis.}

Hierbei wurde das Radium bereits von anderer Seite (Torök und Schein, Goldberg und London, Macintyre, Walk off, S choltz) therapeutisch erfolgreich verwendet. Wir selbst verfügen über 21 Krankheitsfälle verschiedenen Alters und Geschlechts, die zum ersten oder auch wiederholtem Male von der Schuppenflechte befallen wurden. Unabhängig von der Lokalisation und der Ausdehnung der einzelnen Herde wurden stets eine größere Zabl nach mannigfachster Expositionsdauer beobachtet.

Oberflächliche, wenig infiltrierte Herde wurden einer einmaligen Bestrahlung von 3 Min. unterworfen. Am 4. bis 5 . Tage traten die durch das Radium ausgelösten von der Psoriasis-Hyperämie gut unterscheidbaren entzündlichen Reaktionserscheinungen auf. Nach 7 Tagen ging die Reaktion zurück, und gleichzeitig ließ die Schuppung nach. Gegen Ende der zweiten Woche verschwanden die Psoriasiseffloreszenzen. Es restierte eine geringe, wohl auf die Radium-Wirkung zurückzuführende Röte, die auch am Ende der 3. Woche verschwand. Bei den beschriebenen leichteren Formen kam es verschiedene Male bei besonders zarter Haut nach einmaliger Sitzung von 3 Min. nach 7 Tagen zur Blasenbildung, mit deren Abheilung gegen Ende der zweiten Woche auch der Psoriasisherd verschwunden war. Dieselbe Erscheinung bot sich stets bei einer einmaligen Bestrahlung von 10 Minuten. Bei dieser Behandlung trat wiederholt sehr rasch ein Rezidiv auf, das eine nochmalige gleiche Behandlung erforderte, welche dann zu einer endguiltigen $A b-$ heilung führte.

Bei derbinfiltrierten Psoriasisformen führte eine einmalige Sitzung von 10 Minuten am 5. Tage nach auftretender ent- 
Klin., bakt. u. mikr. Bef. b. d. Verw. d. Radiumbromids etc. 431

zündlicher Reaktion zu einem Aufhören der Schuppung. Da nach 8 Tagen die Schuppung sich wieder einstellte, wurde der gleiche Bestrahlungsmodus wiederholt. Auch nach dieser zweiten Bestrahlung kam es zu keiner definitiven Abheilung. Dieselbe wurde erst nach einer dritten gleichartigen Behandlung erreicht.

Recht eigenartig ist ein Fall von Psoriasis, auf den ich hier ausführlicher eingehen will.

Der Patient, ein 27jähriger Photograph, litt neben einer ausgesprochenen Leukopathie seit seinem 17. Lebensjahre an besagter Affektion, die in größeren oder kleineren Zwischenräumen ständig wiederkehrte und unter den üblichen therapeutischen Maßnahmen abheilte. Vor seiner Anfnahme in die Klinik war Patient in einem Krankenhause mit Chrysarobin behandelt worden, das jetzt zum ersten Male eine ungewöhnliche und hochgradige Reizung hervorrief. Im AnschluB an diese Reizung verbielt sich die Psoriasis des Kranken gegen jede therapeutische Maßnahme nicht nur refraktär, die Affektion zeigte sogar einen progressiven Charakter, so daß schließlich ganz große Flächen z. B. fast die ganze vordere Thoraxwand einen gleichmälig erkrankten derbinfiltrierten Herd darstellte.

An Brust und Abdomen wurden nun etwa 60 Stellen einer Bestrahlung von 3 and 5 Minuten unterzogen. Nach ca. 1 Stunde traten die Konturen einer scharf abgegrenzten kreisrunden Rötung auf, welche dem Zentrum der Kapsel entsprach. Dieser Reaktionsherd hob sich nach 12 bis 24 Stunden deutlich über das Niveau der umgebenden Haut und war eingeschlossen von einem $1 / 2 \mathrm{~cm}$ weißen Ring. In den nächsten 3 Tagen steigerte sich die entzündliche Rötung. Nach etwa dreiwöchentlichem Bestand blaßte sie dann allmählich ab. Der früh er schuppende Herd wurde glatt und weiB. An einzelnen Stellen kam es in der zweiten Woche zur Blasenbildung, nach deren Abhebung und Epithelisierung in der 3.-4. Woche gleichfalls vollständige Heilung eintrat. Zirka 6 Wochen später stellte sich an diesen Stellen ein Rezidiv ein, so daß die Wiederholung derselben Prozedur notwendig wurde. An den Ober- und Unterschenkeln, wo die Psoriasis in derselben Weise behandelt wurde, zeigte sich jedoch niemals das gleiche eigenartige Bild. Hier heilte besagte Affektion ebenso ab wie bei anderen Patienten in der früher beschriebenen Weise. - Bei demselben Kranken wurden nun an Brust und Abdomen zahlreiche Stellen so bestrahlt, da zwischen Haut und Kapsel eine drei Millimeter dicke Bleifolie zu liegen kam. Hierbei bot sich dasselbe Bild wie früher. Wurde aber das Präparat in einer. Entfernung von ungefähr einen $1-2 \mathrm{~cm}$ von der Haut auf einem in der Mitte durchbohrten Korkpfropfen die gleiche Zeitdauer appliziert, so heilte jetzt entsprechend der Größe der Kapsel und dem von dem Präparat ausgehenden Lichtkegel (ohne Auftreten des roten Zentrums) mit einem weißen Kreise die Psoriasis ab. Ungefähr 6 Wochen nach der 
Abheilung der einzelnen Stellen an Brust und Abdomen bildeten sich an den bestrahlten Stellen leukodermähnliche zarte weiße Narben, die heute nach 8 Monaten noch unverändert bestehen. Hervorheben möchte ich, daß die leukopathische Hant genan so reagierte, wie normale, pigmentierte.

\section{Carcinom.}

Die von Becquerel, Curie Danysz und Bohn, Apolant, Exner, Plimmer, Hammond am Carcinom gemachten Beobachtungen können wir vervollständigen und bestätigen. Ich verfüge über 19 Fälle (10 Frauen, 9 Männer). In der Mehrzahl handelte es sich um pfennigstück- bis talergroße Cancroide am inneren und äußeren Augenwinkel, Orbitalrand, Nase, an Zunge und Lippen. Auf die Beschreibung jedes einzelnen Falles einzugehen erübrigt sich, da sich mit kaum nennenswerten Differenzen stets dasselbe klinische Bild wiederholt. Alter der Kranken und klinisches Aussehen der Krankheit ließen in keinem Falle einen Zweifel an der Diagnose aufkommen.

Wir haben bei allen Tumoren 4-5tägige Sitzungen von 10-20 Min. angewendet. Fünf Tage nach der ersten Bestrahlung trat die entzündliche Reaktion auf; nach 10-12 Tagen war sie auf der Höhe angelangt. In der Zwischenzeit schmolz der Tumor und vor allem dessen wallartiger Rand zusehends ein. Am 14. Tage war die Reaktion zumeist abgelaufen, und das nunmehr gereinigte und weiche restierende Ulcus, dem der charakteristische Carcinomrand fehlte, epithelisierte sich schnell. In dem einen oder anderen Falle zeigte sich in der Haut nach der Abheilung in der 5.-7. Woche eine schwielige Verdickung, sodaß wir vorsichtigerweise eine nochmalige Behandlung vornahmen.

Nach Auftreten einer oberflächlichen Ulceration (10. bis 12. Tag) heilte die Affektion etwa nach weiteren 5-7 Wochen mit einer glatten weichen Narbe definitiv ab. Ob nicht etwa noch nach sehr langer Zeit Rezidive auftreten, muß die Erfahrung lehren. Wir verfügen zur Zeit nur über eine halbjährige Beobachtungszeit.

Bei 5 Fällen von Cancroid mußten wir die Kapsel in unmittelbare Berührung mit dem Auge selbst bringen. Stets reagierte daon selbst bei geschlossenen Augen die Conjunctiva mit einem mehr oder weniger stärkeren Katarrh, der nach 5 Tagen auftrat, unter feuchten Verbänden aber in einigen Tagen wieder spurlos ablief. In dem einen Falle kam es nach 7 Tagen zu einer oberflächlichen Erosion der Conjunctiva palbr. infer., die jedoch nach 14 Tagen anstandslos abheilte. Eine schädliche Beeinflussung der Hornhaut war in keinem 
Klin., bakt. u. mikr. Bef. b. d. Verw. d. Radiumbromids etc. 433

dieser Fälle zu konstatieren. (Himstedt und $\mathrm{Nagel}$, London, Greeff.) Die dann hierauf angestellten Tierversuche ließen erst nach einmaliger einstündiger Bestrahlung eine hochgradige Keratitis, nach zweistündiger ein Ulcus der Cornea aufkommen.

Daß eine bereits bestehende Drüsenschwellung bei Carcinom keine Kontraindikation für die Behandlung mit Radium ist, wurde von $\mathrm{Exner}$ und Holzknecht bereits beobachtet, und kann von uns nach jeder Richtung hin nur bestätigt werden. In einem besonders eklatanten Falle handelte es sich um ein über kirschengroßes Cancroid der Unterlippe mit gleichzeitiger Schwellung der Submaxillardrüse derselben Seite. Die Drüse, welche nach Abheilung des Tumors kleiner geworden, wurde gleichfalls einer Bestrahlung von dreimal 10 Min. mit zweitägigen Intervallen unterzogen. Es entstand hierbei nach 4 Tagen eine entzündliche Hautreaktion, die spurlos abheilte. Die Drüse selbst war bei der nach 4 Wochen erfolgenden Wiedervorstellung der Patientin nicht mehr nachweisbar. Es muß hier die Frage offen bleiben, ob es sich um eine entzündliche Schwellung oder schon um Metastasenbildung in der Drüse gehandelt. Ex $\mathrm{n}$ er will mehrere Drüsenmetastasen bei Carcinom zum Verschwinden gebracht haben.

So ermutigend alle diese Resultate sind, muß doch ausdrücklich betont werden, daß es bei dieser neuen Behandlungsmethode nicht immer ohne $Z_{w}$ ischenfälle abgeht, wie der folgende Fall lehrt:

Eine 42jährige Patientin litt an einem kleinhandtellergroßen, ganz oberflächlich gelegenen Hautcarcinom der rechten Wange, das in der bekannten Weise mit Radium behandelt wurde. Hierbei nahm nun aber die Affektion, welche bisher einen gutartigen Charakter getragen, eine anscheinend bösartjgere Form an. Unsere therapeutischen Maßnahmen schienen ein schnelleres Wachstum provoziert zu haben; denn aus der Tiefe der Haut erhoben sich zusehends verrucöse Neubildungen, die erst nach Röntgenbehandlung wieder zum Stillstand bzw. Schwund gebracht wurden.

\section{Lupus vulgaris.}

Seit der Halkinschen Mitteilung ist besonders im letzten. Jahre der Lupus vulgaris vielfach erfolgreich mit Radium behandelt worden. (Dantes und Bloch, Macintyre, Delsaux, Davidson, Caspari, Holzknecht, Danlos, Torök und Schein, Hyde and Ormsby, Hallopeau et Gad a ud.) 
Von dem Gedanken der baktericiden Wirkung des Radiums ausgehend, rersuchte $\mathrm{Caspari}$ die Infektionserreger selbst an Ort und Stelle anzugreifen, indem er bei Drüsentuberkulose Radiumlösungen injizierte. Die Versuche sind noch nicht abgeschlossen.

Wir behandelten eine Reihe von Lupusherden der mannigfaltigsten Formen und konnten unsere klinischen Beobachtungen in 17 . Fällen mikroskopisch kontrollieren, wobei die Excisionen in den verschiedensten Stadien nach der Bestrahlung vorgenommen wurden.

Einzelne erbsen- bis 1 Pfennig-Stück große Herde wurden an 4 aufeinander folgenden Tagen 10 Minuten lang bestrahlt. Die gegen Ende der ersten Woche einsetzende entzündliche Röte blaßte in der zweiten Woche allmählich ab. In der 3. bis 4. Woche ließ die Schuppen- und Krustenbildung nach, und in der 6. Woche hatte der früher intensiv braunrote Farbenton einem helleren grauen Platz gemacht. Die Bildung einer Erosion wurde hierbei nicht beobachtet. Die später zur Kontrolle vorgenommene Tuberkulininjektion zeigte eine typische lokale Reaktion.

Ferner wurden 8 Stellen täglich 20 Minuten behandelt. Schon am 5. Tage bildete sich an einzelnen Herden eine Blase, der 2 Tage vorher eine starke entzündliche Röte vorausgegangen war. Am 7. Tage oberflächliche Geschwürsbildung, die nach 14 Tagen sich wieder epithelisierte und mit einer milchweißen Narbe abheilte. Von den früher deutlich sichtbaren Lupusknötchen war jetzt nichts mehr zu erkennen, ebenso wie die wiederholt vorgenommenen Tuberkulinreaktionen stets negativ ausfielen.

Bei 4 Patienten wurden die serpiginösen Ränder größerer Lupusherde mit Radium behandelt, und zwar in der Weise, daß jede einzelne Stelle mit zweitägigen Intervallen $5 \times 15$ Mín. lang bestrahlt wurde. Am 16. Tage hatte die Reaktion in Form einer stark entzündlichen Röte ihren Höhepunkt erreicht, zu der sich 2 Tage später eine mäßige Schwellung und am Ende der 3. Woche eine ganz oberflächliche Erosion gesellte. Mit dem Rückgang dieser Erscheinungen am Ende der 4.-5. Woche reinigte sich der Lupusherd durch Abstoßung der Krusten und Schuppen. In der 7. Woche zeigte die erkrankte Stelle nach Epithelisierung eine kaum erkennbare subkutane Narbe mit grauer livider Verfärbung, die nach mehreren selbst sehr hohen Alttuberkulininjektionen jetzt nicht mehr reagierte.

Wir hatten also mit einer sehr großen Schonung der Gewebe schondieselben guten Resultate zu verzeichnen, die andere Forscher erst nach Auftreten von Ulcerationen beobachteten. 
Klin., bakt. u. mikr. Bef. b. d. Verw. d. Radiumbromids ete. 435

Die langen Applikationszeiten bis zu 120 Stunden, wie sie von französischen $u$. a. Radiotherapeuten vorgeschlagen werden, eignen sich also nach unserer Erfahrung wenigstens, nicht für die Behandlung des Lupus vulgaris.

Die durch die langen Expositionen erzeugten Ulcera brauchen zu ihrer Heilung fast ebenso lange Zeit wie die durch das Röntgen hervorgerufenen. Es ist ja auch gar nicht abzusehen, warum solche tiefen Geschwüre produziert werden sollen. Tuberkelbacillen werden, wie dies aus meinen Tierversuchen hervorgeht, erst in ca. $4 \times 24$ Stunden abgetötet.

Sehr lehrreich erscheint mir ein Fall, der an die unangenehmen Nebenwirkungen nach Röntgenbestrahlungen erinnert und dadurch zur Vorsicht m a b nt. Zwei kaum pfennigstückgroße Lupusherde an der Streckseite des linken Oberschenkels wurden bei einem zwölfjährigen Knaben, der an Knochentuberkulose litt, an fünf aufeinanderfolgenden Tagen je 30 Min. lang mit Radium bestrahlt. Am dritten Tage zeigte sich bereits eine starke entzündliche Röte, die aber scharf auf der behaudelten Stelle lokalisiert blieb; wenigstens schien die Umgebung ganz unbeeinflußt geblieben. Nach dem letzten Behandlungstage kam es zur Blasenbildung, der sich am 8. Tage eine Erosion und am 14. Tage eine Ulzeration anschloß. In der 6 . Woche wurde das torpide und keinerlei Heilungstendenz zeigende Geschwür, das von einer ganz normalen Haut umgeben war, im Gesunden und bis tief auf die Fascie excidiert und der Defekt durch Nähte geschlossen. Nach Entfernung des ersten Verbandes ergab sich am achten Tage folgender Befund: Die Schnittwundränder waren gut adaptiert und bereits ziemlich fest verklebt. Von den Stichkanälen aber gingen zentrifugal fortschreitende dem anfänglichen Ulcus identische Ulzerationen a us. Die Umgebung der Schnittwunde zeigte dieselbe Verfärbung. Im Verlauf der nächsten 5-6 Tage vereinigte sich die von den Stichkanälen ausgehenden Ulzerationen zu einem etwa 5 markstückgroßen typischen Radiumulcus, das im Verlauf der nächsten Woche gleichfalls wieder unverändert und ohne Neigung zu heilen bestehen blieb. Einige in der Nähe nicht mit Radium behandelte excidierte Stellen heilten per primam ab. Es wurde etwa in der 4 . Woche nach der 1. Excision eine Transplantation nach Thiersch ohne Erfolg vorgenommen. Frst nach tiefer Excision des Ulcus und Transplantation mit gestieltem Lappen heilte das Ulcus ab.

\section{Verrucae durae.}

Auch diese Affektion wurde bei 3 Patienten erfolgreich mit Radium behandelt, wobei 10 Warzen an den 
Händen zum Verschwinden gebracht wurden. Nach dreitägigen Dosierungen zu je 10 Minuten war noch kein Erfolg zu verzeichnen. Eine viertägige Behandlung zu je 20 Minuten genügte aber, um die papillären Exkreszenzen obne irgend welche entzündliche Reaktion zum Verschwinden zu bringen. Nach ihrer Abstoßung nahm die jetzt glatte Warze im Laufe der folgenden Woche eine entzündliche Röte und Schwellung an, an die sich in der dritten Woche eine Ulzeration anschloß, unter der die Warze in 8 Tagen einschmolz. Eine weiße Narbe deutet jetzt auf ihren früheren Sitz hin. Wurde viermal in zweitägigen Intervallen zu je 15 Minuten bestrahlt, so trat am 7. Tage eine entzündliche Röte mit leichter Schwellung auf. Die Warze selbst verschwand mit dem Abklingen der Reaktion ungefähr nach 14 Tagen. Am Ende der 4. Woche ließ sich eine kaum erkennbare subkutane Narbe konstatieren.

\section{Sycosis vulgaris.}

Diese Affektion, der wir therapeutisch so of machtlos gegenüberstehen, konnte auch durch Radium nicht nachhaltig beeinflußt werden (T o r ök und Schein). In 3 von uns behandelten Fällen handelte es sich um eine ausgebreitete Knötchen- und Pustelbildung im Bereiche des Bartes. Die entzïnd lich infiltrierte, sehr empfindliche Haut schien auf das Radium eher und stärker zu reagieren, als wir sonst $u$. a. pathologischen Verhältnissen zu sehen gewöhnt waren. Schon eine einmalige Sitzung von 3 Minuten zeitigte am 2. Tage heftige entzündliche Erscheinungen, die sich von der bereits bestehenden bläulichroten Verfärbung der Haut deutlich als hellrote Herde unterscheiden ließen. Diese entzündliche Reaktion blieb bis Ende der 3. Woche bestehen, ohne daß dann mit ihrem Abklingen in der 4.- $\overline{5}$. Woche irgend eine auf Abheilung hinweisende Veränderung an dem Herd selbst eingetreten wäre. Noch stärkere Reaktionen zu erzeugen, schien schon deshalb nicht ratsam, weil wir dann erfahrungsgemäß. Narbenbildung erwarten mußten. Daß wir eine baktericide Wirkung auf die Erreger der Sycosis vulgaris ausüben würden, war nach den von uns zu gleicher Zeit ausgeführten bakteriologischen Untersuchungen nicht zu erwarten.

\section{Trichophytie.}

Zwei Fälle, lokalisiert am Halse und an der Streckseite des rechten Unterarmes.

Bestrahlt wurden die serpiginösen Ränder hier täglich $5 \times 15$ Minuten, dort $5 \times 30$ Minuten. Im ersteren Falle schloß 
Klin., bakt. ฉ. mikr. Bef. b. d. Verw. d. Radiumbromids etc. 437

sich an die Behandlung am 5. Tage eine entzündliche Rötung und Schwellung, in dem andern am 8. Tage bereits eine oberflächliche Erosion an. Die darauf abgetragenen Schuppen zeigten stets auf Maltose-Agar ein reiches Wachstum der Pilze. Mit dem Abblassen der entzündlichen Reaktion und dem Rückgang der Erosion trat keine Veränderung an dem Herde selbst ein.

\section{Favus.}

Behandelt wurde nur ein Fall mit Lokalisation am Kopf in fünftägigen Sitzungen zu 10 Minuten, die vollständig ergebnislos ausfielen. Die Kopfhaut selbst reagierte nicht.

Von weiteren therapeutischen Maßnahmen nach dieser Richtung hin wurde Abstand genommen, da die zu gleicher Zeit gemachten bakteriologischen Untersuchungen bewiesen, dal die Erreger dieser Affektion erst in ca. 3 Tagen abgetötet werden, also zu einer Zeit, wo eine erfolgreiche Behandlung zugleich mit einer schweren Alteration der Gewebe verknüpft sein mübte.

Um eine Übersicht über unsere Resultate und die von uns angewandte Dosierung $\mathrm{zu}$ ermöglichen, habe ich die nachstehende Tabelle zusammengestellt. Aber nochmals betone ich, daß es falsch wäre, wollte man in ihr eine MaximaldosenTabelle sehen, an die sich jeder Nachprüfende schematisch zu halten hat. Fraglos liegen hier die Dinge ebenso wie bei der Röntgentherapie, wo man seinen Apparat kennen muß, wenn anders man unter gleichen Bedingungen gleich gute Resultate erzielen will.

Um jedoch eine Nachprüfung meiner Versuche zu ermöglichen, verweise ich auf die S. 14 verzeichnete Tabelle, mittels der an der Hand eines Chromoradiometers nach Holzknecht auch ungleichwertige Präparate mit einander verglichen werden können, wenn nur die Expositionszeiten entsprechend modifiziert werden. 


\begin{tabular}{|c|c|c|c|}
\hline & Behandlungszeit & Reaktion & Abheilung \\
\hline $\begin{array}{l}\text { Lichen ruber } \\
\text { planus }\end{array}$ & $3-4 \times 10-15 \mathrm{Min}$ & $\begin{array}{c}\text { Nach } 4-5 \text { Tagen } \\
\text { Rötung, Schwellung } \\
\text { (Erosion) }\end{array}$ & Nach 2-3 Wochen \\
\hline $\begin{array}{c}\text { Lupus } \\
\text { erythenaat. }\end{array}$ & $\begin{array}{l}3 \times 5 \text { M. } 3 \times \text { Wiederh } \\
\text { nachAblaufd.Reakt. }\end{array}$ & $\begin{array}{l}\text { II. Woche entzündl. } \\
\text { Rötung }\end{array}$ & 7. Woche \\
\hline $\begin{array}{c}\text { Naevi } \\
\text { vasculosi. }\end{array}$ & $\underset{\text { Pausen. }}{4 \times 5 \text { Min. in } 2 \text { tägl. }}$ & $\begin{array}{l}\text { II.-III. Woche ent- } \\
\text { zündliche Rötung }\end{array}$ & 4.-(6.) Woche \\
\hline $\begin{array}{c}\text { Naevi } \\
\text { pigmentosi. }\end{array}$ & $\begin{array}{l}4 \times 5 \text { Min. }(-10 \text { Min.) } \\
\text { ev. Wiederholung }\end{array}$ & $\begin{array}{l}14 \text { Tage entzündl. } \\
\text { Rötung }\end{array}$ & $\begin{array}{l}\text { 3.-4. Woche } \\
\text { (event. Narbe) }\end{array}$ \\
\hline $\begin{array}{c}\text { Acne } \\
\text { rosacea. }\end{array}$ & $\begin{array}{c}4 \times 10 \text { Min. in 2tägig. } \\
\text { Pausen. }\end{array}$ & $\begin{array}{ll}\text { II.-III. Woche } & \text { Wochtung } \\
\text { entzündl. Rötun }\end{array}$ & $\begin{array}{l}\text { 4.-(5.) Woche } \\
\text { (event. Narbe.) }\end{array}$ \\
\hline Psoriasis. & $1-2 \times 5-10$ Min. & $\begin{array}{l}\text { 5. Tag. Abstoßung } \\
\text { der Schuppen }\end{array}$ & I.--II. Woche \\
\hline Carcinom. & $\begin{array}{l}4-5 \times 10-20 \text { Min. } \\
\text { ev. Nachdosierung }\end{array}$ & $\begin{array}{c}\text { II. WocheEinsuhmel. } \\
\text { des Tumors }\end{array}$ & (4.)-5. Woche \\
\hline $\begin{array}{l}\text { Lupus } \\
\text { vulgaris. }\end{array}$ & $\begin{array}{l}\text { 3-4 } 4 \text { 10-15Min.in } \\
\text { 2t. Paus., ev. Nachd. }\end{array}$ & $\begin{array}{l}\text { 8-14 Tagen; unter } \\
\text { entz. Schwellung } \\
\text { Reinigung d. Herdes }\end{array}$ & $\begin{array}{l}\text { (4.) -5. Woche } \\
\text { (event. Narbe) }\end{array}$ \\
\hline $\begin{array}{c}\text { Verrucae } \\
\text { durae. }\end{array}$ & $3-4 \times 15-20 \mathrm{Min}$ & $\begin{array}{l}8 \text { Tagen, } \\
\text { Abstoßung }\end{array}$ & $\begin{array}{l}\text { (3.) -4. Woche } \\
\text { (Narbe) }\end{array}$ \\
\hline $\begin{array}{l}\text { GefäßBektas. } \\
\text { a. Röntgenn. }\end{array}$ & $5 \times 20$ Min. & $\begin{array}{c}\text { Erosion } \\
\text { in 14 Tagen }\end{array}$ & 4 Wochen: Narbe \\
\hline
\end{tabular}

\section{Bakteriologische Untersuchungen.}

Daß den Radiumstrahlen eine baktericide Wirkung zukommt, ist eine bekannte Tatsache. Ausführliche Mitteilungen liegen von A s chkinass und Caspari, Pfeiffer und Friedberger, Hoffmann, Danysk und Scholtz vor. Die ersteren konnten die von den $\mathrm{Curies}$ bereits gemachte Beobachtungen bestätigen, wonach von den vom Radium ausgehenden zwei Strahlungsarten nur die stark absorbierbaren einen keimschädigenden Einfluß ausüben, die aber im Gegensatz zu den anderen, Luftschichten von mehr als $4 \mathrm{~cm}$ Länge nicht mehr zu durchsetzen vermögen. 
Klin., bakt. u. mikr. Bef. b. d. Verw. d. Radiumbromids etc. 439

Pfeiffer und Friedberger konnten feststellen, daß Typhusbazillen, die in einer Entfernung von $1 \mathrm{~cm}$ nach 48 Stunden abgetötet wurden, bei 6 bis $10 \mathrm{~cm}$ Distanz vollständig unbeeinflußt bheben. Cholerabazillen starben in unmittelbarer Berührung mit dem Radium nach 16 Stunden, Milzbrandsporen an Seidenfäden nach $3 \times 24$ Stunden $a b$.

Scholtz, der mit eben demselben Präparate arbeitete, kam bei seinen Untersuchungen mit Typhusbazillen und Staphylokokken zu ganz ähnlichen Resultaten. Ihm gelang es auch im Gegensatz zu $\mathrm{H}$ offma n $\mathrm{n}$ Bakterien in Flüssigkeiten abzutöten.

Danysz glaubte auf Grund seiner zahlreichen Versuche nur an eine Entwicklungshemmung der Bakterien; boi wenigen wie besonders bei Milzbrandbazillen konnte er eine Abtötung konstatieren.

Zuerst und am eingehendsten haben sich Aschkinas und Caspari in ihrer Arbeit: "Über den Einfluß dissoziierender Strahlen auf organisierte Substanzen, insbesondere über die bakterienschädigende Wirkung der Becquerelstrahlen" mit dieser Frage beschäftigt und festgestellt, daß die Wachstumshemmung, bzw. Abtötung der Bakterien nicht auf eine Schädigung der Nährböden, sondern auf direkte Beeinflussung der Parasiten selbst zurückzuführen ist.

Ich habe nun bei meinen Versuchen die Technik und Anordnung der genannteu Autoren im wesentlichen beibehalten, nur wurden meine Beobachtungen nicht nur an $\operatorname{Pro}_{0}$ digiosus, Staphylokokken, Streptokokken und Tuberkelbazillen, sondern auch an den Erregern der Trichophytie und des Favus angestellt.

Meine Versuchsanordnung gestaltete sich folgendermaßen:

I. Die Bakterien wurden indirekte Berührung mit der in einem sterilen Kondom befindlichen Radiumkapsel gebracht.

II. Die Bakterien wurden innerhalb einer luftdicht verschlossenenPetrischale in einer Entfernung von ca. $1 \mathrm{~cm}$ bestrahlt.

III. Zwischen Kapsel und Bakterien befand sich Glas,

a) indem die Kapsel auf der Nährbodenseite der Schale lag,

b) indem die Kapsel auf dem Deckel der Schale oder außen an einem Reagenzglas fixiert wurde.

Diese Versuche wurden in allen Fällen mehrfach wiederholt, um Fehlerquellen nach Möglichkeit auszuschalten. Die 
Differenzen zwischen den einzelnen Versuchsreihen sind so gering, daß sie kaum merklich ins Gewicht fallen. Die Stundenzahlen entsprechen ununterbrochenen Expositionszeiten.

Zur Prüfung des erzielten Effektes wurden dann die Bakterien auf ihnen besonders zusagende Nährböden verimpft. Eine Eintrocknung wurde nach Möglichkeit verhindert. Die Tuberkelbazillen, deren volle Virulenz vorher durch Inokulationen von Meerschweinchen (Tod nach 3 Wochen) festgestellt war, wurden in Bouillonaufschwemmungen zu je $1 \mathrm{~cm}^{3}$ intraperitoneal oder intravenös auf Meerschweinchen weiter übertragen.

Die Streptokokken, deren Virulenzgrad vorher festgestellt $\left(1 \mathrm{~cm}^{3}\right.$ Bouillonaufschwemmung tötet intravenös ein Kaninchen in ca. 3 Tagen), wurden nach der Bestrahlung wieder in Bouillon suspendirt und einem Kaninchen intravenös injiziert $\left(1 \mathrm{~cm}^{3}\right)$. Ein Teil wurde auf Ascites-Agar verimpft.

\section{Tabelle I.}

Diese Versuchsreihe bezieht sich auf die unter I und II gemachten Beobachtungen und ist, da sie keine wesentlichen Unterschiede zeigt, in dieser einen Tabelle untergebracht.

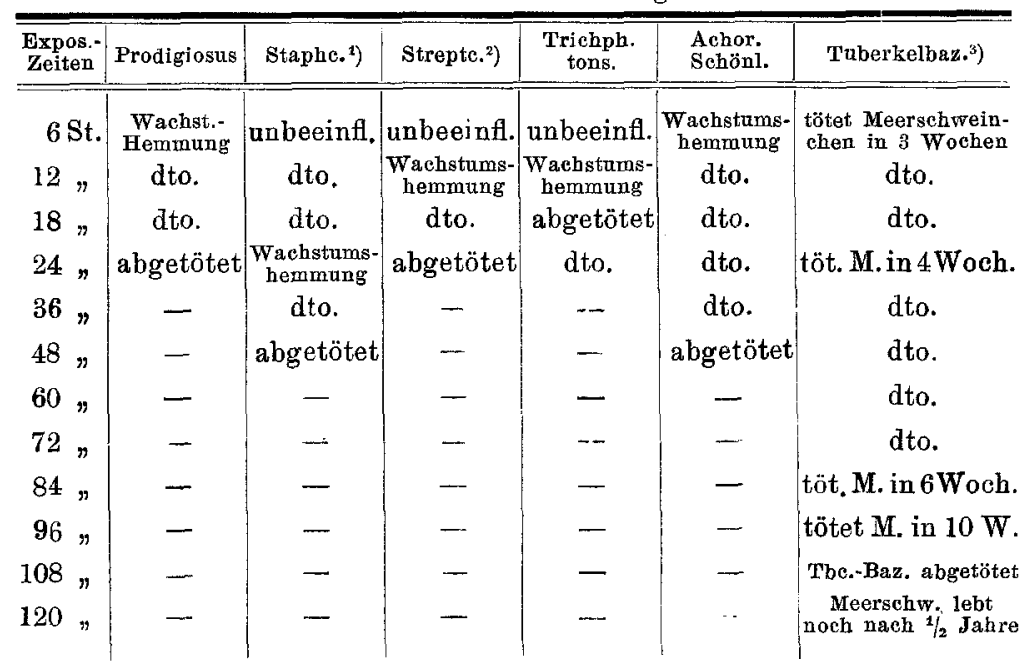

1) Der Pustel einer sehr hartnäckigen Sycosis vulg. entnommen.

2) Aus einem Erysipel stammend.

3) Gezüchtet aus dem Spatum eines Phthisikers. 
Klin., bakt. u. mikr. Bef. b. d. Verw. d. Radiumbromids etc. 441

Tabelle II.

Diese Tabelle, welche die Versuche III $a$ ) und $b$ ) enthält, ergibt geringe Differenzen zur vorhergehenden. Ein besonderes Interesse gewinnt sie dadurch, als sie uns zeigt, daß Glas doch bis zu einem gewissem Grade hemmend auf die Radiumstrahlen einwirkt. Es ist diese Tatsache insofern von Bedeutung, als einzelne Radiotherapeuten ihre Kristalle in Glas einschmelzen.

\begin{tabular}{|c|c|c|c|c|c|c|}
\hline $\begin{array}{l}\text { Exposs- } \\
\text { Zeiten }\end{array}$ & Prodigiosus & Staphe. & Strepte. & Trich. tons. & Ach.Schönl. & Tuberkelbaz. \\
\hline $6 \mathrm{St}$. & unbeeinfl. & unbeeinfl. & unbeeinfl. & unbeeinf. & unbeeinfl. & $\begin{array}{l}\text { tötet Meersehw, in } \\
3 \text { Wochen }\end{array}$ \\
\hline $12 n$ & $\begin{array}{c}\text { Wachstums- } \\
\text { hemmung }\end{array}$ & dto. & $\begin{array}{l}\text { Wachstums- } \\
\text { hemmung }\end{array}$ & dto. & dto. & dto. \\
\hline $18 \%$ & dto. & dto. & dto. & $\begin{array}{c}\text { Wachstums- } \\
\text { hemmung }\end{array}$ & $\begin{array}{c}\text { Wachstums- } \\
\text { hemmung }\end{array}$ & dto. \\
\hline 24 , & dto. & dto. & dto. & dto. & d to. & dto. \\
\hline 36 & abgetötet & dto. & abgetötet & abgetötet & dto. & dto. \\
\hline 48 " & dto. & $\begin{array}{l}\text { Wachstums } \\
\text { hemmung }\end{array}$ & - & - & dto. & dto. \\
\hline $60 "$ & ato. & abgetötet & - & - & abgetötet & dto. \\
\hline $84 "$ & - & - & - & - & - & töt. M. in 7Woeh \\
\hline $96 "$ & - & - & 一 & -. & - & dto. \\
\hline $108 \eta$ & - & - & - & - & - & Tbc. abgetötet \\
\hline $120 "$ & - & - & - & - & -- & $\begin{array}{l}\text { Meersehw. lebt noch } \\
\text { naeh über } 1 / 2 \text { Jahre }\end{array}$ \\
\hline
\end{tabular}

Mikroskopische Befunde.

Den mikroskopisehen Untersuchungen liegen 26 Excisionen zu Grunde, von denen 10 normaler Haut, der Rest zumeist Lupus vulgaris, und auch einem naevus vasculosus angehören. Um die Übersicht zu erleichtern und Wiederholungen zu vermeiden, gebe ich zuerst eine Beschreibung der an gesunder Haut gemachten Befunde.

1. Nach einer einmaligen Expositionsdauer von $10 \mathrm{Min}$. und in fast gleicher Weise von $20 \mathrm{Minuten}$ sind $12 \mathrm{Stunden}$ später, abgesehen von einer stärkeren Füllung der Gefäße, die sich klinisch als leichte entzündliche Röte kund gibt, mikroskopisch keine Veränderungen an den Geweben nachweisbar.

Nach 24 Stunden bietet das Präparat dasselbe Bild.

Nach 48 Stunden, also zu einer Zeit, wo die behandelte Stelle als hellroter Kreis sich von der Umgebung scharf abhebt, sehen wir mikroskopisch eine akute Entzündung.

In der ganzen Höhe des Coriums, wie im Stratum subcutaneum sind die Gefäße strotzend mit Blut gefüllt. Die Kapillaren des Papillarkörpers sind deutlich erweitert. Die Gefäße weisen bereits Scheiden von ausgewanderten Leukozyten auf, unter denen vereinzelte Mastzellen zu beobachten sind. weisen.

An den Gewebszellen lassen sich keine Veränderungen nach- 
Am 4. bis 6. Tage sind die entzündlichen Erscheinungen noch ausgesprochener. Das perivaskuläre Bindegewebe zeigt ein dichtes Leu. kocyteninfiltrat, welches auffallend zahlreich Mastzellen enthält. Vor allem aber fällt jetzt eine neue Veränderung auf, die sich in der Intima der $G$ efä $B$ e abspielt: die Endothelzellen sind vergrößert, heben sich, bläschenförmig aufgetrieben, von der Gefäßwand $a b$ und ragen in das Lumen hinein. Auch ihre Kerne haben an Umfang zuge nommen. einflußt.

Bindegewebszellen und Epidermis sind noch vollständig unbe-

Erst acht Tage nach der Bestrahlung macht sich auch im Epithel die Wirkung des Radiums geltend, ebenso wie an den Bindegewebszellen.

Gleich den Endothelien sind die Zellen des Coriums und der Epidermis gequollen und haben eine ungleichmäßige kugelige Form angenommen. An einzelnen Stellen läßt sich Vacuolenbildung nachweisen. Am intensivsten scheinen die basalen Epithelien getroffen zu sein. Die Epidermis ist im ganzen aufgelockert, die Spalträume zwischen ihnen erweitert, in die sich hier und da Leukocyten hineindrängen. Nach dem verhornten Epithel zu, das seine normale Beschaffenheit behalten hat, nehmen die beschriebenen Degenerationserscheinungen allmählich ab.

Am 12. Tage beobachten wir ein Statium, das wir klinisch unter dem Bilde der Blasenbildung kennen lernten und dadurch zustande kommt, daß das entzündliche Ö̉dem die Hornschicht und die oberen Epithelschichten von den unteren abhebt. Dieses Stadium ist als eine Vorstufe der Erosion anzusehen, die etwa

am 14. Tage auftritt. Die oben beschriebene Vacuolenbildurg in den gequollenen Epithelien greift immer mehr um sich. Der Kern wird nach der Peripherie gedrängt und nimmt selbst milgestaltete Formen an. Deutlich kann man schrittweise diese einzelnen Phasen verfolgen. Ein Teil der Zellen geht schließlich zu Grunde, für welcke Tatsache die allenthalben zerstreuten Kernreste beweisend angesehen werden müssen.

Diese Degenerationserscheinungen führen schl ießlich zum Zustande einer Erosion. Wie in den Schichten der Epidermis sehen wir auch an den Bindegewebszellen des Coriumsund ebenso am Drüsenund Follikelepithel die gleichen Alterationen.

Die Anhäufung von Leukocyten und Mastzellen hat noch größere Dimensionen angenommen, und infolge der schweren Alteration der Gefäße beobachten wir Hämorrhagien. Elastische Fasern bleiben hierbei anscheinend unbeeinflußt; wenigstens läßt sich durch mannigfach angestellte Färbemethoden nach dieser Richtung hin keine Degenerationserscheinung nachweisen. Ebenso zeigte sich das Kollagen unbeeinflußt. Eine Ulzeration tritt bei dieser Bestrahlungsdauer nicht auf.

Die Befunde an Ulzerationen wurden an einem durch einmalige einstündige Bestrahlung am 5. Tage zur Ulzeration gebrachten und an diesem Tage excidierten Stücke erhoben. 
Das kraterförmig in die Tiefe reichende Ulcus ist von einem undifferenzierbaren gelblichen Belage bedeckt, in dem sich einzelne Leukocyten und schwachgefärbte kernlose Epithelzellen nachweisen lassen. Fibrinfärbung negativ. Sodann folgt in der Ungebung eine dichte Infiltrationsschicht aus Plasmazellen und polynucleären Leucocyten. Diese Schicht sendet längs der Gefäße einzelne Infiltrationszapfen in die Tiefe. - Die elastischen Fasern sind in den dem "Ulcus" zunächst liegenden Schichten fast vollständig zu Grunde gegangen, die Gefäße nur durch einzelne elastische Faserringe und durch Blutpigmentanhäufung angedeutet. Die Bindegewebszellen sind zu einer fast homogenen transparenten schlecht färbbaren Masse aufgequollen. Mehr nach der Umgebung zu lassen sich wieder die oben bereits beschriebenen Zelldegenerationen wiedererkennen.

Diese Beschreibung gilt auch für die nach einer Bestrahlung von $6,12,24,48$ und mehr Stunden, so daß es sich erübrigt, auf sie näher einzugehen. Es handelt sich hier stets um immer tiefer greifende Zerstörungen und Geschwürsformen, die event. bis in das Fettgewebe hineinreichen.

Stellen wir nun diesen Präparaten von normaler Haut die Befunde an Lupus vulgaris in den entsprechenden Bestrahlungsstadien gegenüber, so beobachten wir überall die gleichen Degenẹrationserscheinungen, die sich zuerst am Gefäßapparat und dann an den übrigen Gewebselementen abspielen. Natürlich sind die an sich schon ausgesprochenen Erscheinungen der entzündlichen Infiltration noch vermehrt. Von einer spezifischen Beeinflussung des $L$ up usknötchens ist aber nichts zu erkennen.

Wurde die Behandlung bis zur Ulzeration ausgedehnt, sehen wir ein der Expositionsdauer entsprechend tiefes kraterförmiges Geschwür, dem, wie alle andern zelligen Elemente, auch die Tuberkel zum Opfer gefallen sind. Andererseits sind dieselben in unmittelbarster Nähe des Ulcusrandes ganz unverändert geblieben.

LetztereBefundewarenesaugenscheinlich, dieHalkin zu der Ansicht brachten, daß das Radium in der Therapie des Lupus vulgaris keine besondere Verwendung finden könne, da es auf pathologische Prozesse nur soweit seinen EinfluB ausübe, als es die Gewebe vernichte.

Daß diese Anschauungen indes unrichtig sind, davon überzeugen uns Präparate, die 3-4 Wochen nach der Abheilung a uch nicht u l zerierter Stellen excidiert worden waren, bei denen es vielmehr n u r z u einer lebhaften reaktiven Entzündung gekommen war, und die entweder ohnejegliche Narbenbildung oder höchstens mit einer zarten subkutanen Narbe abgeheilt waren. Der Befund ist folgender:

Die oben beschriebene Alteration der Intima führt zur Gerinnung des Blutes in den Kapillaren (Arterien und Venen), es bildet sich ein Thrombus. Es kommt dann zu einer vollständigen Organisation im Gefäß. 
lumen, so daß schließlich an Stelle des Gefäßes ein Strang von jungen Bindegewebszellen tritt, dem einige elastische Faserringe und Längszüge als Rest der früheren Gefäßwand beigemengt sind.

In den größeren Gefäßen, deren Intima abgeblättert ist, finden sich stellenweise ähnliche Thrombosierungen. Die Muscularis ist nicht alteriert. Die Adventia, an der wir in den früheren Stadien massenhafte Anhäufung von Leukocyten beobachteten, kommt es zu einer allmählichen Organisation des Infiltrates. Die ganze Gefäßwand erscheint dadureh verdickt, und die Bindegewebswucherung führt schließlich zu einer totalen Verlegung des Gefäßlumens.

In den früheren Stadien konnten wir beobachten, wie die Leukocyten sich mit Vorliebe auch um die Riesenzellen anhäufen. In gleicher Weise, wie bei den Gefäßen kommt es nun auch hier an Stelle des Infiltrates zu einer Bindegewebsneubildung und schließlich zur vollständigen Abkapselung des Lupusknötchens, so daB dieses dadurch wahrscheinlich unschädlich gemacht wird. In etwa 5 Wochen nach $\mathrm{Ab}$ heilung der reaktiven Entzündung gemachten Excisionen tritt n u $\mathbf{n}$ a $\mathbf{c} \mathrm{h}$ eine Veränderung des Knötchens selber auf. Es kommt zur Vacuolenbildung und Zerfall der Riesen-Granulations- und epitheloiden Zellen und damit zur allmählichen Resorption des Knötchens.

\section{Es ist also nicht nötig, es zu tiefen Ulzerationen kommen zu lassen, sondern es genügt, durch den oben angeführten $\mathrm{Be}$ - strahlungsmodus lediglich eine lebhafte reaktive Entzündung herbeizuführen.}

Die elastischen Fasern werden durch das Radium gar nicht beeinflußt. In tiefen Ulzerationen, also bei einer vollständigen Einschmelzung des Gewebes sehen wir noch Reste der Fasern. Erst, wenn es zur Abheilung, also zur Narbenbildung gekommen, wird ihre Anordnung eine ungleichmäßige dadurch, daß sie an einzelnen Stellen fehlen, an anderen zu dichten Kräueln geballt liegen, ein Befund, wie wir ihn fast bei jeder Narbenbildung zu sehen gewöhnt sind.

Im übrigen vermochten wir trotz der verschiedensten Färbemethoden anch in diesen Stadien keine für Radiumwirkung spezifischen Veränderungen nachzuweisen.

Außer Lupus habe ich ein Stückchen eines $\mathrm{Naevus} \mathrm{vasculosus}$ untersuchen können. Hier konnten wir die Gefäßveränderungen besonders gut verfolgen. Es handelt sich um ein oberflächlich gelegenes Feuermal des Armes, das nach der Behandlung mit Radium mit einer milchweisen Narbe abgeheilt war.

Entsprechend der Größe des Epitheldefektes zeigte sich die Epidermis verdünnt, das Epithel selbst aber sonst unveràndert. Im Corium stieben wir auf die uns aus den früheren Beschreibungen bekannte subkutane Narbenbildung. Die GefäßIumina blutleer oder thrombotisch verlegt.

Den Einfluß der Beequerelstrahlen a uf $\mathrm{C}$ ancroide mikroskopisch zu untersuchen, blieb uns versagt, da uns die Fälle nur unter der Be- 
dingung zur Verfügung gestellt wurden, daß wir von jedem chirurgischen Eingriff absehen.

Als Resüme der mikroskopischen Befunde möchte ich noch einmal hervorheben, daß die ersten nachweisbaren Veränderungen am Gefäßapparat sich abspielten und die Reaktionen am Epithel erst in 2. Linie in Frage kommen; konnten wir doch in der Tiefe des Coriums bereits augenscheinliche Prozesse beobachten, die selbst zur Narbenbildung führten, ohne daß das Epithel nachweislich alteriert war. Nach intensiveren Expotionen machen sich allerdings auch an $\mathrm{ihm}$ in nachhaltigster Weise die Degenerationserscheinungen bemerkbar.

Zum Schluße möchte ich mir erlauben, meinem hochverehrten Chef, Herrn Geheimrat Prof. Dr. Neisser, für die liebenswürdige Anregung und Unterstiutzung bei dieser Arbeit meinen ehrerbietigsten Dank auszusprechen.

\section{Literatur.}

Apolant, H. Deutsche med. Wohhenschr. 1904. Nr. 13. pag。 455. Über die Einwirkung der Radiumstrablen auf das Carcinom der Mäuse. - Aschkinass. Verhandl. d. Ges. d. Naturf, a. Ärzte. 1901. Hamburg. II. T. p. 467. - Aschkinass und Caspari. Arch. f. d. ges. Physiol. Bd. LXXXVI. 1901. p. 603. Über den Einfluß dissoziierender Strahlen auf organische Substanzen; insbesondere über die bakterienschädigende Wirkung der Becquerelstrahlen. - A s chkinas s. Pflügers Archiv. 1901. Becquerel und Carie. Comptes rendus 1901. Bd. CXXXII. p. 1289. Bohn. Comptes rendus 1903. Bd. CXXXVI. p. 1012, 1013. 1085, 1086. I d e m. Arch. gen. med. 1903. 48. - Bulkley. Duncan. Lupus of Cheek Treated by Radium. The journ. of cutan. diseases. 1904. Bd. XXII. Nr. 2. pag. 91. - Caspari. Berl. klin. Wochensehr. 1903. p. 768. Zeitschr. für diät. u. phys. Therapie. 1904/05. Bd. VIIr. - Crzellitzer. Berl. klin. Wochenschr. 1903. Nr. 28. pag. 650. Becquerelstrahlen und Blindheit. Dantes und Bloch. Ann. de dermat. et syph. 1901. pag. 986. Note sur le traitement du Lupus erythemateux par des applications de Radium. Danlos. Ann. de dermat. et syphil. 1902. I. III. - Danys z. Comptes rendus 1903. Bd. CXXXVI. Académie des sciences. Sitzung vom 16. und 23./II. 1903. Münchn. med. Wochenschr. 1903. Nr. 13. p. 584. Pathogene Wirkung der vom Radium ausgehenden Strahlen und Emanationen auf verschiedene Gewebe und Mikroorganismen. - Delsanx. La presse otolaryngologique, belge 1903, 8. Kef. Münchn. med. Wochenschr. 1903. 48. p. 2120. Vorläufige Mitteilung übèr die Behaudlung des Lupus der oberen Luftwege mittelst Radium. - D a vids o n. Brit. medic. journal. 1903. Exner. Münchn. med. Wochenschr. 1903. Nr. 28. p. 1237. Behandlung von Neugebilden mit Radiumstrahlung. Wiener klin. Wochenschr. 1903. 27. K. k Ges. d. Ärate in Wien. 26. Juni 1903. - Giesel, F. Ber. der d. chem. Ges. XXXII. Bd. H. 19. Über radioaktive Stoffe. Chem. Berichte 
1900. Band XXIII. - Goldberg und Lond on. Derm. Zeitschr. 1903. Bd. X. 5. - Greeff. Dtsch. med. Wochenschr. März 190t. Nr. 13. Über Radiumstrahlen and ihre Wirkung auf das gesunde und blinde Auge. Halkin. Arch. f. Derm. 1903. Bd. LXV. Über den Einfluß d. Becquerelstrahlen auf die Haut. - Hall o pea a et Gadaud. Ann. de Dermat. et Syph. I. III. 1902. - $\mathrm{H} \mathrm{a} \mathrm{m} \mathrm{m} \mathrm{o} \mathrm{nd.} \mathrm{Radium} \mathrm{gegen} \mathrm{Ulcus} \mathrm{rodens,} \mathrm{(Brit.}$ med. Journ. Nr. 2260.) Deutsche med. Wochenschr. 1904. Nr. 20. p. 749. - Hartigan. Series of cases treated by Radium. The Brith. Journ. of Derm. 1904. Bd. XVI. Nr. 3. p. 105 . - v. Henri et Meyer, A. C. R. de la Soc. de Biol. 1903. Nov. p. 1414. Folia haematologica 1904. Nr. 2. p. 112. - He in eke. Münch. med. Wochenschr. 1903. Nr. 48. pag. 2090. Über die Einwirkung der Röntgenstrablen auf Tiere. - Himsted und Nage l. Wiener klin. Wochensehr. 1902, -- Ho f fm a nn. Hyg. Rundschau. 1903. Nr. 18. - Hol z knecht, G. Münch. mediz. Wochenschrift. 1903. Nr. 41. p. 1798. K. k. Ges. d. Ärzte in Wien. Juni 1903. - Idem. Wien. klin. Wochenschr. 1903. 27. - I d e m. Kongr. d. derm. Ges. in Serajevo. 1903. - Holaknecht und Schwarz. Münch. med. Woch. 1903. Nr. 27. p. 1189. Über Radiumstrahlen. - Hyde and $0 \mathrm{rmsby}$. D. med. Woch. 1903. Nr. 5. - Jum on. Journ. de malad. cut. et syph. 1903. 11. p. 854. Le Radium. Propiétés physiques et thérapentiques. - London. Berliner klin. Woch. 1903. Nr. 23. p. 523. Münch. med. Wochenschr. 1903. Nr. 24. p. 1043. Zur Lehre von den Becquerelstrahlen und ihren physiologischpathologischen Bedeutungen. - Ma cin ty re, J. Brit. med. journ. 1903. p. 199. Juli. Die therapeutische Wirkung der Radiumsalze. - Miethe. Berl. klin. Wochenschr. Nr. 51. p. 1180. Über das Radium. - Morton. Röntgen- und Radiumstrahlen. (Brit. med. Journ. Nr. 2260.) D. medizin. Wochenschr. 1904. Nr. 20. p. 749. - Neisser, A. Intern. derm. Kongr. iu Serajewo 1903. - Pf eiffer, R. und Friedberger. Berliner klin. Woch. 1903. Nr. 28. pag. 640. Wiener klin. Woch. 1903. Nr. 16. Münch. med. Woch. 1903. Nr. 29. p. 1269. Über bakterientötende Wirkung der Radiumstrahlen. - Perthes. D. med. Wochenschr. Nr. 17. April. 1904. p. 632. Versuche über den Einfluß der Röntgen- und Radiumstrahlen auf die Zellteilung. - Plimmer. Krebsbehandlung mit Radiumbroncid. Deutsche med. Woch. 1904. Nr. 19. pag. 717. - Scholtz, W. Deutsche med. Woch. 1904. 3. p. 94. Über die physiologische Wirkang der Radiumstrahlen und ihre therapeutische Verwendung. - Soddy, Fr. München. med. Wochensch. 1903. Nr. 39. p. 1694. Die Behandlung der Phthise mit Radium- und Thoriumstrahlen. - Török und Schein. Pester medizin. chir. Presse. 1902. - W alk off. Photogr. Rundschau. 1900. 14. p. 189. Weber. Deutsche med. Woch. Nr. 13. März 1904. pag. 457. Über unsere beutige Kenntnis der Radioaktivität. 